\title{
Uncoupling of ribosome biogenesis and Tor activation by TRIM-NHL proteins promotes terminal differentiation
}

\author{
Jinghua Gui ${ }^{1}$ and Felipe Karam Teixeira ${ }^{*}$ \\ ${ }^{1}$ Department of Genetics, University of Cambridge, Downing Street CB2 3EH, Cambridge, UK. \\ *Corresponding author: felipe.karam-teixeira@gen.cam.ac.uk
}

\begin{abstract}
Proper stem cell differentiation relies on a balance between cellular growth and terminal differentiation, but the mechanisms coordinating these processes remain elusive. Recent studies indicate that ribosome biogenesis ( $\mathrm{RiBi}$ ) and protein synthesis, two of the most energy-consuming cellular processes supporting growth, are tightly regulated and yet can be uncoupled during stem cell fate transitions ${ }^{1-6}$. Here, using the Drosophila adult female germline stem cell (GSC) and larval neuroblast (NB) systems, we show that Mei-P26 and Brat ${ }^{7}$, two Drosophila TRIM-NHL paralogues of the mammalian TRIM32 protein family, are responsible for uncoupling RiBi and protein synthesis during GSC and NB differentiation, respectively. This is achieved by TRIM-NHL-mediated activation of the Target of rapamycin (Tor) kinase and concomitant repression of RiBi specifically during stem cell differentiation. In consequence, the anabolic reprogramming established by TRIM-NHL activity creates the conditions for terminal differentiation. In agreement with this, depletion of mei-P26 or brat, which results in excessive cellular growth and defective terminal differentiation ${ }^{8-10}$, can be counterbalanced by ectopic activation of Tor together with suppression of RiBi, allowing completion of differentiation. Our work indicates that TRIM-NHL proteins uncouple RiBi and translation activities to coordinate growth and differentiation, and proposes that the control of cellular resources provides a meter for terminal differentiation.
\end{abstract}


Coordination of RiBi and protein synthesis is essential for the regulation of cell growth, proliferation, stress response, and cell survival ${ }^{2,11}$. In many in vivo and in vitro stem cell systems, microscopic, biochemical, and molecular analyses have revealed that stem cells often feature translation and $\mathrm{RiBi}$ activities that are significantly different from their immediate differentiating progenies ${ }^{3,4,12}$. Specific examples include the adult germline stem cells, the intestinal stem cells, and the larval neuroblasts in Drosophila ${ }^{5,8,13,14}$, as well as a variety of mouse adult stem cells in the hematopoietic, neural, muscle, and skin systems ${ }^{1,2,6,15,16}$. Detailed measurements of ribosomal RNA synthesis, nucleolar volume, ribosome content, and protein synthesis rates indicate that translation and $\mathrm{RiBi}$ activities are dynamically regulated during fate transitions, with genetic and pharmacological manipulations revealing that their tight control is critically important for tissue homeostasis, preventing over-proliferation and protecting self-renewal or differentiation capacities $^{2,3,5,6}$. Even though the dynamic changes of translation and RiBi are pervasive and critical during stem cell differentiation, the mechanisms by which these are regulated remain to be determined ${ }^{17}$.

The Drosophila ovary presents an ideal in vivo system for dissecting the regulation of $\mathrm{RiBi}$ and protein synthesis during differentiation. In this system, Germline stem cells (GSCs) ${ }^{18}$, found attached to the somatic niche at the anterior-most part of the ovaries, show lower translational activity and remarkably higher $\mathrm{RiBi}$ rates in comparison to neighboring differentiating cells. Upon asymmetric cell division and niche exclusion, the differentiating cystoblast (CB) undergoes four rounds of divisions with incomplete cytokinesis before terminally differentiating into a 16-cell cyst (cc), a developmental transition that is characterized by higher translation and lower RiBi (Extended Data Fig. $1 \mathrm{a})^{5,10}$. Experimental modulation of RiBi and protein synthesis activities during germline differentiation has been shown to affect the balance between selfrenewal and differentiation, resulting in either premature loss of GSCs, block in differentiation, or tumorigenesis ${ }^{5,10,14}$. Given the role of the evolutionary conserved Target of rapamycin (Tor) kinase in coordinating these activities to promote cell growth ${ }^{19}$, we set out to investigate whether Tor activity is regulated during GSC fate transition. To do this, we first took advantage of an antibody against the phosphorylated form of the ribosomal protein S6 (pS6), a downstream target and readout of the activity of the Tor pathway ${ }^{20,21}$.
Immunofluorescence microscopy analysis revealed that GSCs were devoid of p-S6 $(n=117)$, but signal was detectable from the differentiating $\mathrm{CB}$ stage onwards (Fig. 1a, b; Extended Data Fig. 1a). While only $\sim 12 \%$ ( 5 out of 41 ) of CBs were positively marked by $\mathrm{p}-\mathrm{S} 6$, all $(\mathrm{n}=37$ ) 2-cell and $\sim 93 \%$ (81 out of 87) of 4-cell cysts were p-S6 ${ }^{+}$, with the penetrance of p-S6 signal acutely declining in 8 -cell $(\sim 59 \%$; 45 out of 76) and terminally differentiated 16 -cell cysts $(\sim 11 \%$; 8 out of 74$)$. Analysis using the cell-cycletracing FUCCI system ${ }^{22}$ confirmed that p-S6 expression was not detected in GSCs, regardless of the cell-cycle phase, but present in cysts throughout the cell cycle (Extended Data Fig. 1b-d). Moreover, in vivo analysis revealed that a short incubation of adult ovaries with rapamycin, a specific inhibitor of the Tor kinase, was sufficient to abolish the p-S6 signal in differentiating cells (Fig. 1c). As the protein levels for Tor and the Target Of Rapamycin Complex 1 (TORC1) co-factor Raptor are constant from GSCs to 16-cell cysts (Extended Data Fig. 1e), our results indicate that Tor activity is regulated during GSC differentiation.

Differentiating germ cells are characterized by a strong increase in translation ${ }^{5}$, which coincides with Tor activation. To test whether this increase in translation is mediated by Tor, we measured global protein synthesis rates in vivo by using immunofluorescence and O-propargyl-puromycin (OPP), which is incorporated into nascent polypeptides $^{5,23}$. Robust OPP incorporation in differentiating cells was abolished by rapamycin treatment (Fig. 1c, d) ${ }^{5}$. These results demonstrate that Tor activation underlies the increase in translation observed during cyst development and coincides with the stages of differentiation during which RiBi is downregulated $^{5,10}$.

Nucleolar volume, a proxy for $\mathrm{RiBi}$, has been shown to be negatively regulated during GSC differentiation by Mei-P26, the TRIM-NHL protein ortholog of the mammalian TRIM32 family of proteins ${ }^{10}$.

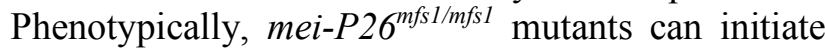
the GSC differentiation program, but differentiating cysts featuring enlarged nucleoli and increased cellular volume are unable to complete terminal differentiation (Fig. 2a, b) ${ }^{10}$. Surprisingly, our analysis revealed that differentiating cysts were devoid of p-S6 signal in mei-P2 $6^{m f s / m f s l}$ mutants, suggesting that Mei-P26 is required for Tor activation during differentiation (Fig. 2b, d). In agreement with this, the increase in global translation rate that is observed in differentiating cysts was abolished in $m e i-P 26^{m f s l} / m f s l$ mutants (Fig. $2 \mathrm{c}, \mathrm{e}$ ). 


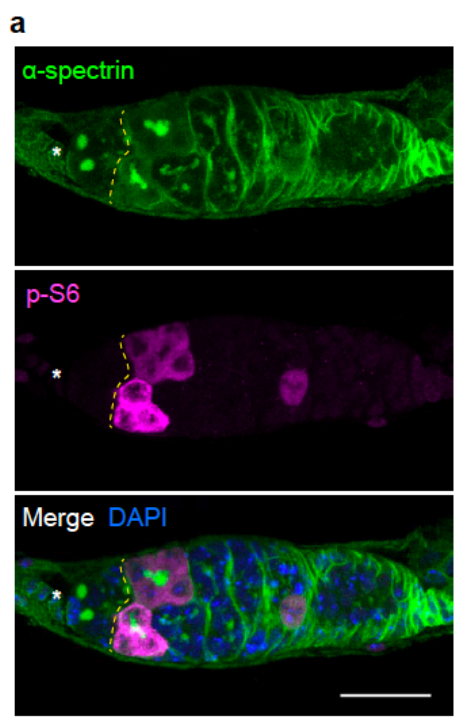

b

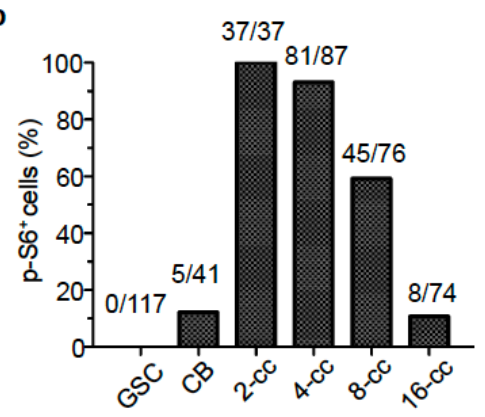

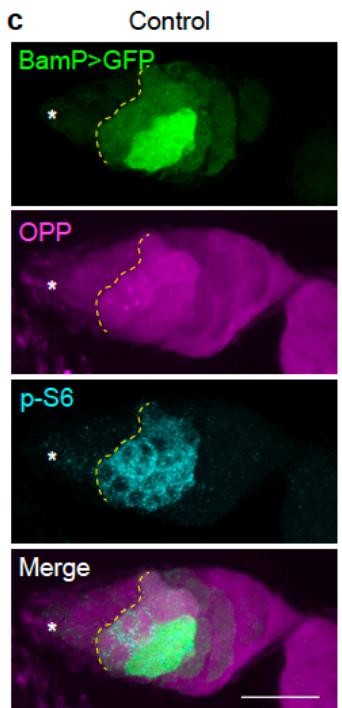

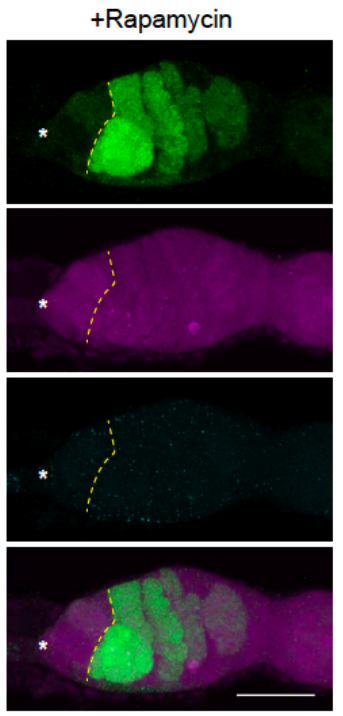

d

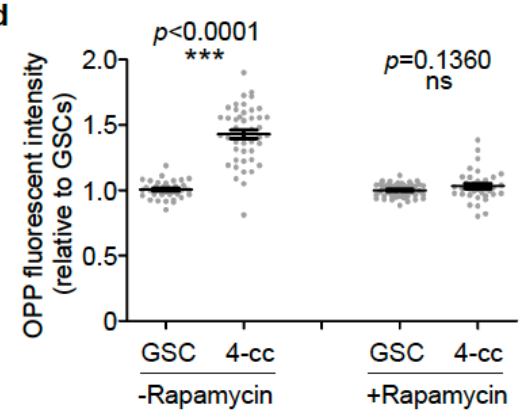

Figure 1. Tor regulates translation rate during germline stem cell differentiation.

a, Representative image of a germarium labeled with $\alpha$-spectrin (spectrosomes/fusomes, green), p-S6 (Tor activity, magenta), and DAPI (nuclei, blue). b, Distribution of $\mathrm{p}-\mathrm{S} 6^{+}$cells in different stages of germline differentiation. $\mathbf{c}$, Representative germaria, with the differentiation marker BamP $>$ GFP (green), labeled with OPP (translation rate, magenta) and p-S6 (Tor activity, cyan) with or without rapamycin treatment. d, OPP fluorescent intensity measurements of germaria with or without rapamycin treatment. Data are mean \pm s.e.m (d). ${ }^{* * *} p<0.0001$, t-test. Asterisks indicate the GSC niche. Dashed lines depict the boundary between GSCs and differentiating cells (a, c). Scale bars, $20 \mu \mathrm{m}(\mathbf{a}, \mathbf{c})$.

To determine whether Tor activity is directly promoted by Mei-P26, we overexpressed mei-P26 in wild-type germ cells, which resulted in $>75 \%(187$ out of 249) of GSCs showing ectopic p-S6 signal (Fig. 2f, g). In addition, extensive p-S6 signal was detected in fully differentiated egg chambers in mei-P26 overexpressing ovaries (Extended Data Fig. 2a). Interestingly, sustained mei-P26 overexpression induces premature GSC differentiation, eventually leading to loss of germ cells ${ }^{10}$. Experiments conducted with food supplemented with rapamycin revealed that Tor inhibition not only abolished p-S6 signal in mei-P26 overexpressing ovaries but was also sufficient to suppress the GSC loss phenotype (Fig. 2f, g; Extended Data Fig. 2a, b), indicating that ectopic Tor activation underlies Mei-P26-dependent premature loss of GSCs. To characterized the effects of modulating mei-P26 expression in GSCs, we coupled overexpression and downregulation of meiP26 with a loss-of-function mutation for the differentiation factor Bag-of-marbles $(\mathrm{Bam})^{24}$, which completely blocks GSC differentiation. Immunofluorescence analyses revealed that OPP incorporation was positively correlated with mei-P26 levels in bam $^{\Delta 86}$ GSC-like cells while confirming that nucleolar volume displays an inverse correlation with mei-P26 expression (Fig. 2h-j; Extended Data Fig. $2 \mathrm{c})^{10}$. Indeed, RNA-sequencing experiments revealed that RiBi-related genes - including those encoding ribosomal proteins, nucleolar markers, and ribosomal RNA (rRNA) polymerases - were negatively regulated by Mei-P26 (Extended Data Fig. 2d). Altogether, our results demonstrate that Mei-P26 promotes Tor activity and protein synthesis while inhibiting $\mathrm{RiBi}$, uncoupling two of the most important anabolic processes during germline differentiation. 

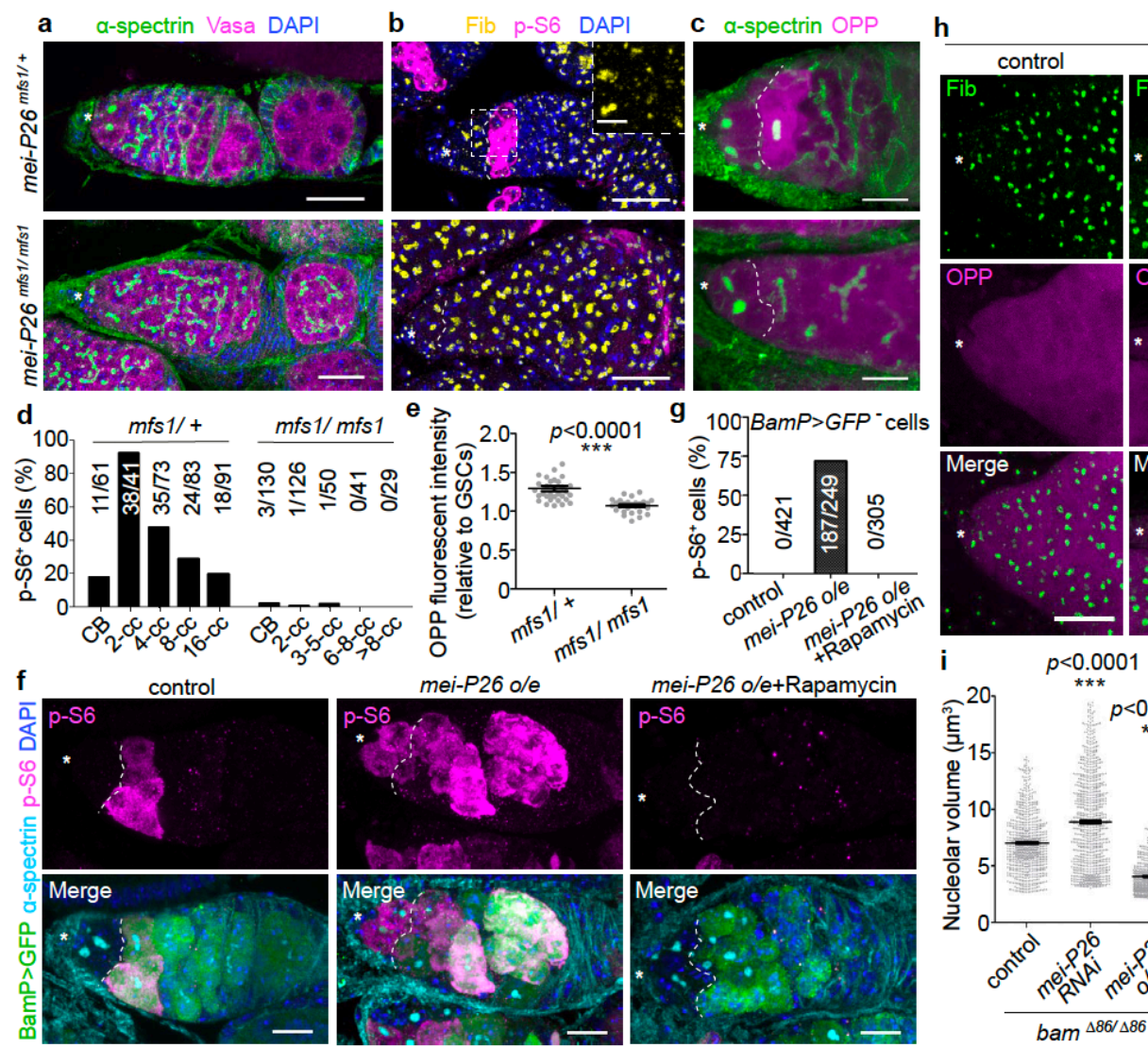

bam $^{\Delta 86 / \Delta 86}$
mei-P26 RNA

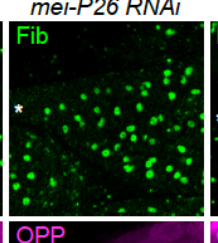

mei-P26 o/e
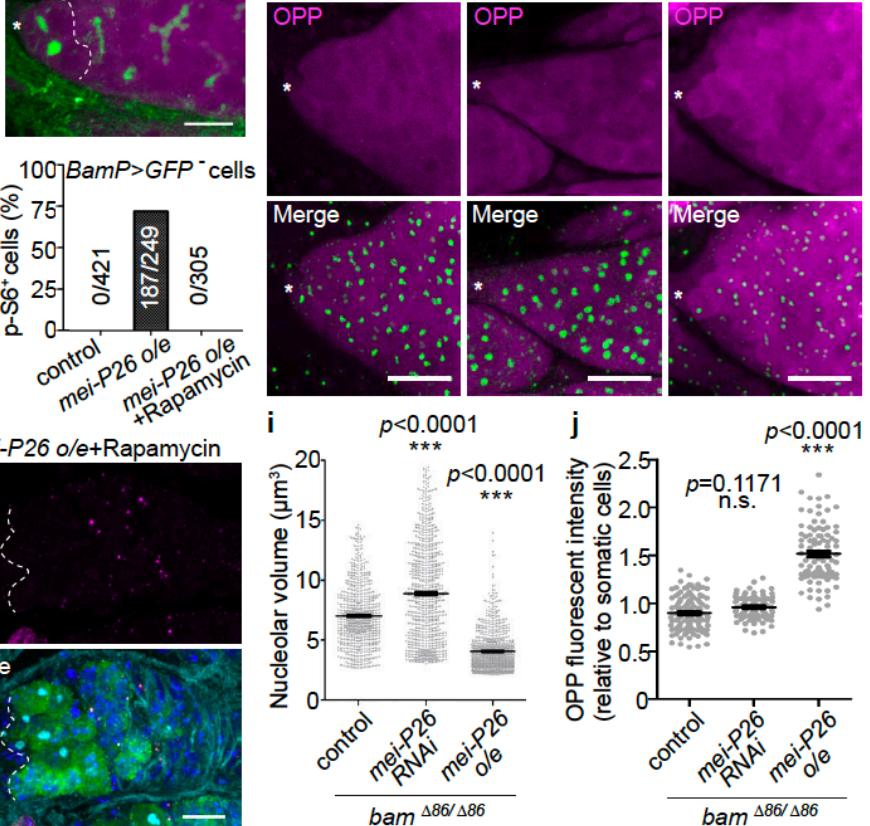

Figure 2. Mei-P26 activates Tor kinase during GSC differentiation, uncoupling protein synthesis and RiBi.

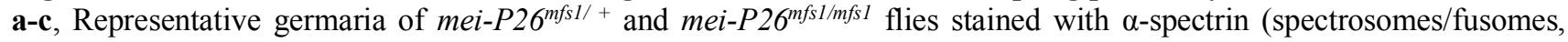
green, a, c), Vasa (germline, magenta, a), DAPI (nuclei, blue, a, b), Fib (nucleoli, yellow, b), p-S6 (Tor activity, magenta, b), and OPP (translation rate, magenta, c). d, Distribution of $\mathrm{p}-\mathrm{S} 6^{+}$cells in different stages of germline differentiation from

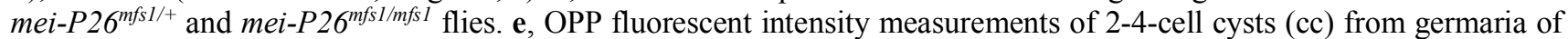

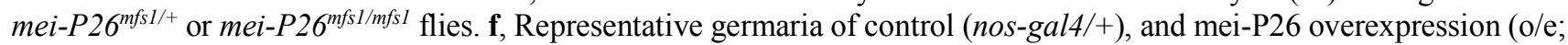
nos-gal4/UASp-mei-P26) flies with or without rapamycin feeding, stained with p-S6 (magenta), $\alpha$-spectrin (cyan), GFP (BamP $>$ GFP, differentiating cells, green) and DAPI (blue). g, Proportion of p-S6 ${ }^{+}$GSCs in germaria of control (nosgal4/+), and mei-P26 overexpression (o/e; nos-gal4/UASp-mei-P26) with or without rapamycin feeding. h, Representative germaria of control (nos-gal4/+), mei-P26 knockdown (nos-gal4/UAS-mei-P26 RNAi) or mei-P26 overexpression (o/e; nos-gal4/UASp-mei-P26) in the bam $^{\Delta 86 / \Delta 86}$ background, stained with Fib (green) and OPP (magenta). i and j, Measurements of nucleolar volume (i) and translation rates (j) in GSC-like cells $\left(\right.$ bam $\left.^{486}\right)$ in germaria of mei-P26 knockdown (nosgal4/UAS-mei-P26 RNAi) or mei-P26 overexpression (o/e; nos-gal4/UASp-mei-P26) in the bam ${ }^{486 / 486}$ background. Data are mean \pm s.e.m. ${ }^{* * *} p<0.0001$, t-test $(\mathbf{e}, \mathbf{i}, \mathbf{j})$. Asterisks indicate the GSC niche. Dashed lines depict the boundary between GSCs and differentiated cells (a-c, f). Scale bars, $20 \mu \mathrm{m}(\mathbf{a}, \mathbf{b}, \mathbf{f}, \mathbf{h}), 10 \mu \mathrm{m}(\mathbf{c})$, or $5 \mu \mathrm{m}(\mathbf{b}$, inset).

To determine how pervasive the regulation of Tor activity by TRIM-NHL is during stem cell differentiation, we turned our focus to the larval neuroblast system where the Brain Tumour (Brat) paralog of Mei-P26 promotes differentiation while downregulating RiBi activity during neurogenesis ${ }^{8,25}$. In this system, Brat activity is restricted to the differentiating progeny by disproportionate segregation into the differentiating daughter cell during the asymmetric division of the type-II $\mathrm{NBs}^{8,9}$. As in the case of GSCs, NBs are characterized by a larger cell size and a higher RiBi rate compared to the differentiating progeny, which include the immature intermediate neural progenitors (INPs), the mature INPs, and the ganglion mother cells (GMCs) ${ }^{8}$. Immunofluorescence analyses in wild-type larval brains indicated that robust p-S6 signal is developmentally modulated, being consistently detected in NBs and mature INPs $\left(\mathrm{Dpn}^{+}\right)$but rarely observed in GMCs or differentiated neurons $\left(\mathrm{Pros}^{+}\right)$ in the Type-II NB lineage (Fig. 3a, b; Extended Data Fig. 3). This situation is similar to the one we observed in GSCs and led us to test whether Brat is required for Tor activation during NB differentiation. To do this, we used brat RNAi driven by the NBspecific driver insc-Gal4 ${ }^{26,27}$. Our results revealed that 
a
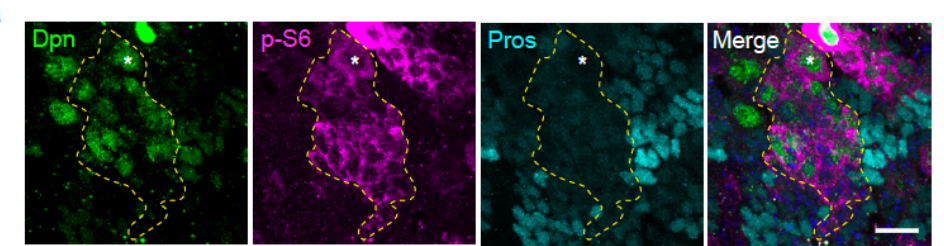

b
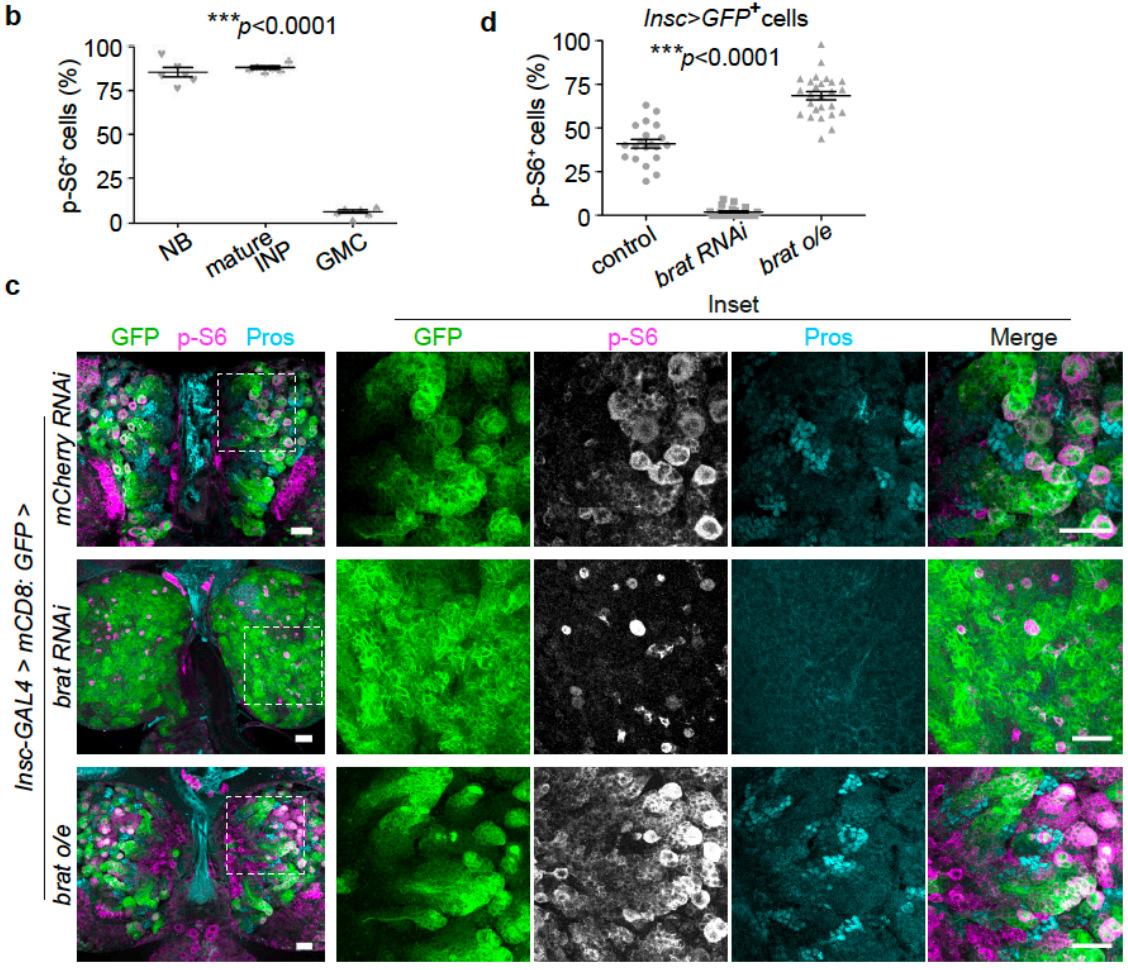

Figure 3. Brat is required for Tor activation during type-II NB differentiation.

a, Representative confocal images of type-II NB lineages stained with Dpn (self-renewal marker, green), p-S6 (Tor activity, magenta), and Pros (pro-differentiation marker, cyan). Dashed lines outline the $\mathrm{p}-\mathrm{S} 6^{+}$area of a type-II NB lineage. Asterisks indicate type-II NBs. $\mathbf{b}$, Distribution of $\mathrm{p}-\mathrm{S}^{+}$cells in different stages of type-II NB differentiation. $\mathbf{c}$, Representative larval brains of control ( $m$ Cherry RNAi), brat RNAi or brat overexpression (o/e) driven by insc-GAL4, stained with GFP (insc $>m C D 8: G F P$, green), p-S6 (magenta or grey) and Pros (cyan). White dashed rectangles (left) indicate the sources of the insets (right). d, The percentage of $\mathrm{p}_{-} \mathrm{S}^{+} \mathrm{GFP}^{+}$in control (mCherry RNAi), brat RNAi knockdown, and brat overexpression (o/e) larval brains. Data are mean \pm s.e.m. ${ }^{* * *} p<0.0001$, one-way ANOVA (b, d). Scale bars, $10 \mu \mathrm{m}(\mathbf{a})$ or $25 \mu \mathrm{m}(\mathbf{c})$.

the p-S6 signal was mostly abolished in brat deficient brains (Fig. 3c, d), becoming restricted to a small number of cells, whose number and distribution resemble that of NBs in wild-type brains. To directly test whether Brat is sufficient to promote Tor activation, we characterized the expression of p-S6 in larval brains where we overexpressed brat. In this context, the p-S6 expression domain was robustly and ectopically expanded into GMCs and newly differentiated neurons (Fig. 3c, d). These results demonstrate that, as in the case of GSC differentiation, the Mei-P26 paralog Brat activates Tor while downregulating $\mathrm{RiBi}$ during differentiation ${ }^{8,25}$.
Given that mei-P26 and brat mutants display defective differentiation, leading to the overaccumulation of proliferative cells with enlarged cellular volume (Fig. 2-3) (10 $^{8-1}$ we investigated whether the misregulation of Tor activity and RiBi are responsible for the observed phenotypic changes. To do so, we modulated Tor and RiBi activities in meiP26 and brat depleted animals using genetic and pharmacological approaches. While brat knockdown led to the accumulation of partially differentiated $\mathrm{Dpn}^{+}$Pros $^{-}$cells that lack any sign of differentiation, increased Tor activity through overexpression of Raptor in a brat RNAi context (brat RNAi, raptor: $H A$ ) was sufficient to decrease the $\mathrm{Dpn}^{+} \mathrm{Pros}^{-}$ population and to promote NB differentiation $\left(\mathrm{Dpn}^{-}\right.$ 


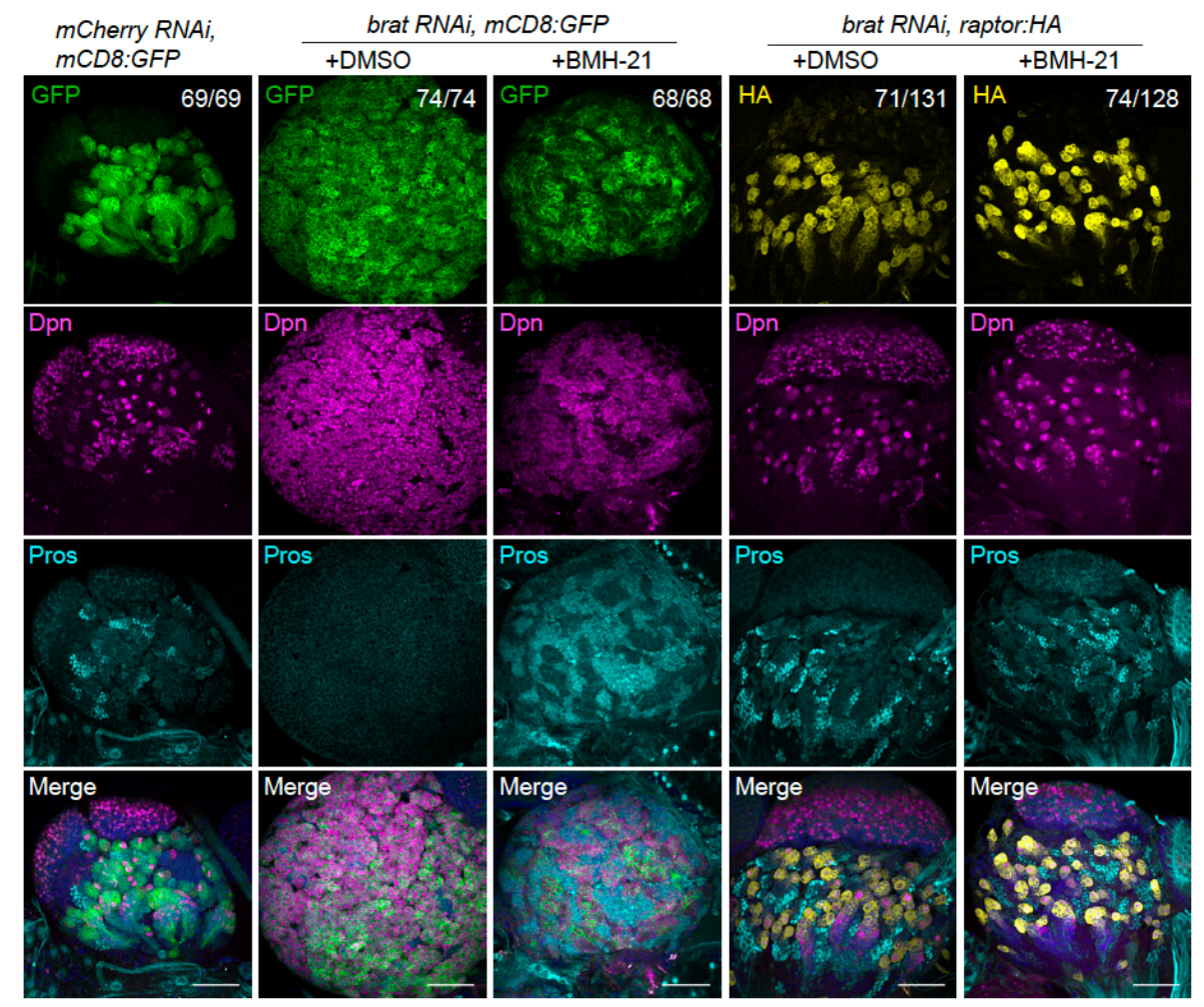

Figure 4. Modulation of RiBi or Tor activity is sufficient to promote terminal differentiation in brat larval brains. Representative larval brain lobes of control ( $m$ Cherry RNAi, $m C D 8: \mathrm{GFP})$, brat knockdown (brat RNAi, $m C D 8: \mathrm{GFP})$, and brat knockdown with raptor overexpression (brat RNAi, raptor:HA) flies (driven by insc-GAL4) with or without BMH-21 feeding. Samples were stained with GFP (mCD8, green) or HA (Raptor, yellow), Dpn (self-renewal marker, magenta), Pros (pro-differentiation marker, cyan), and DAPI (nuclei, blue). The numbers in the upper panel indicate the penetrance of the phenotype. Numbers in the top panel indicate the penetrance of phenotypes out of three independent experiments. Scale bars, $50 \mu \mathrm{m}$.

Pros $^{+}$cells; Fig. 4). Indeed, in brat RNAioverexpressing Raptor larvae, $\sim 50 \%$ of brain lobes had no apparent phenotypic abnormalities, recapitulating a similar distribution of cellular markers when compared to control brains ( $m C D 8: G F P$; Fig. 4, Extended Data Fig. 4a, b). This cellular rescue resulted in a functional recovery: climbing assays conducted with adult flies revealed that while brat-deficient flies had reduced mobilities, adult flies overexpressing Raptor in a brat RNAi context were indistinguishable from the control flies (Extended Data Fig. 5a, b). In addition, clonal analyses using the rrat $^{11}$ mutation revealed that knockdown of the Tor-antagonizing regulators Tsc 1 or Tsc2 was sufficient to restore NB differentiation in the absence of Brat in $11 \%$ of clones ( $t s c 1$ RNAi or tsc2 RNAi, Extended Data Fig. 4c). These results show that Tor activation is sufficient to promote the differentiation of brat-deficient NBs.

To determine whether inhibition of RiBi has a similar effect on the differentiation of brat-deficient NBs, we fed larvae with BMH-21, a specific inhibitor of RNA Polymerase I (PolI) activity that leads to a reduction of rRNA synthesis ${ }^{28}$. In this context, brat RNAi brains showed partial differentiation, with the nuclear translocation of the differentiation marker Pros and downregulation of the undifferentiated $\mathrm{Dpn}^{+}$ population (Fig. 4; Extended Data Fig. 6a, b). In this case however, the distribution of cellular markers did not fully recapitulate the wild-type pattern and we observed numerous cells positive for both Pros and Dpn markers, suggesting that in this case bratdeficient cells differentiated without experiencing the canonical program of type-II NB differentiation (Extended Data Fig. 6b). Although we did not observe a synergistic effect of feeding larvae with BMH-21 and simultaneously overexpressing Raptor in brat RNAi brains (Fig. 4), our results underscore the role of Tor activation and RiBi suppression in promoting terminal differentiation in NBs and suggest a partial redundancy between Tor activation and RiBi inhibition. 

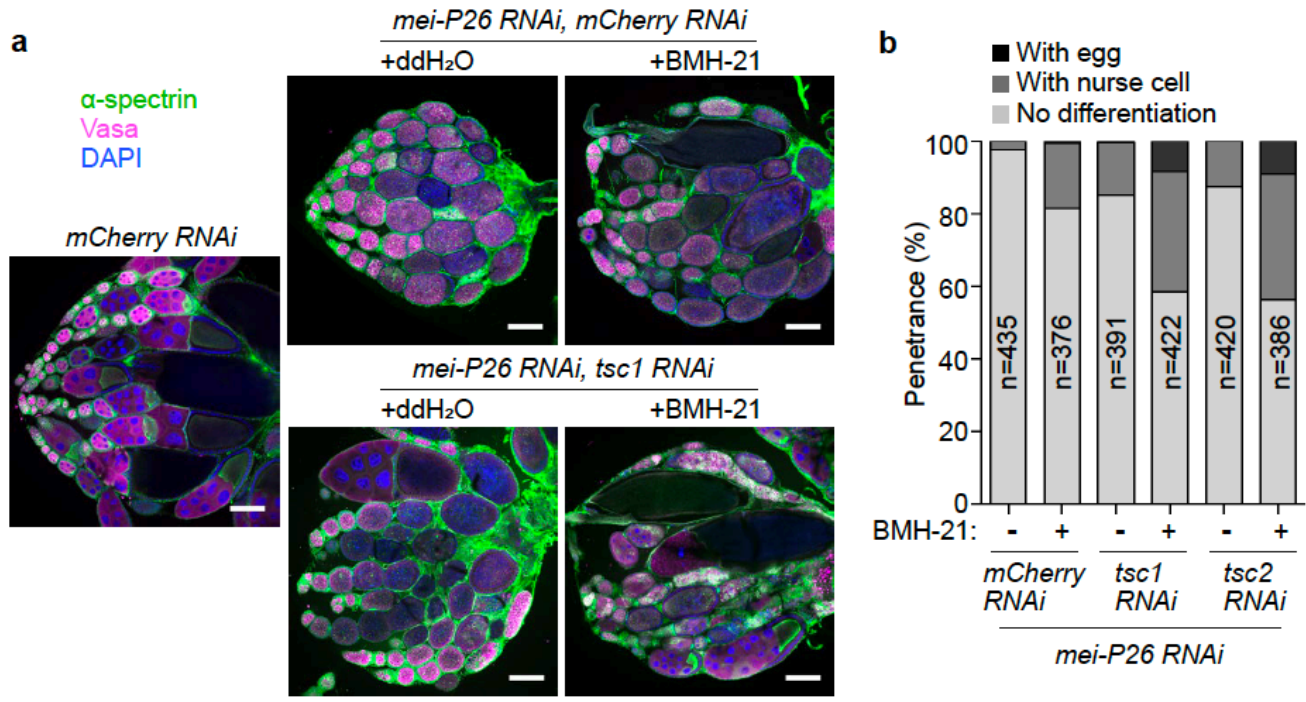

Figure 5. Simultaneous changes in RiBi and Tor activity promote terminal differentiation in mei-P26 ovaries.

a, Representative ovaries of control (mCherry RNAi), mei-P26 mCherry double knockdown (mei-P26 RNAi, mCherry $R N A i$ ), and mei-P26 tscl double knockdown (brat RNAi, tscl RNAi) flies, with or without BMH-21 feeding. Samples were stained with $\alpha$-spectrin (spectrosome/fusomes, green), Vasa (germline, magenta), and DAPI (nuclei, blue). b, Penetrance of ovary phenotypes of control (mCherry RNAi), mei-P26 mCherry double knockdown (mei-P26 RNAi, mCherry RNAi), mei-P26 tsc1 double knockdown (mei-P26 RNAi, tsc1 RNAi), and mei-P26 tsc2 double knockdown (mei-P26 RNAi, tsc1 $R N A i)$ with or without BMH-21 injection. $n$ indicates the number of ovarioles analyzed. Scale bars, $200 \mu \mathrm{m}(\mathbf{b})$.

To determine whether similar interactions could be observed in the germline, we investigated whether modulating Tor and RiBi activities could also circumvent the mei-P26 RNAi-mediated block to differentiation. Using the mei-P26 germline RNAi as a background, we observed that treating adult flies with BMH-21 (mCherry RNAi + BMH-21) or genetically activating Tor during germline development by knocking down Tsc1 and Tsc2 (tsc1 RNAi or tsc2 RNAi) led to a small suppression of the mei-P26-induced block in differentiation (Fig. 5a, b). While the ovaries of such flies were consistently filled with partially differentiated cysts similar to mei-P26 RNAi controls, terminally differentiated egg chambers were observed in $15-20 \%$ of the ovarioles. However, when both treatments were combined, robust terminal differentiation was observed in $\sim 40 \%$ of the cases, including $\sim 10 \%$ of ovarioles being filled with fully developed eggs (Fig. 5a, b). Collectively, our results demonstrate that TRIM-NHL proteins, by activating Tor and concomitantly suppressing RiBi, uncouple the two most energy-consuming biosynthetic activities during differentiation, and consequentially restrict growth and promote terminal differentiation.

The Tor pathway is known to orchestrate many anabolic processes, such as RiBi and translation, to coordinate the build-up of resources to sustain cellular growth ${ }^{19}$. During stem cell differentiation however, $\mathrm{RiBi}$ and protein synthesis rates are actively regulated, and these dynamic changes are essential for properly balancing self-renewal, growth, and differentiation. Here, we have shown that the TRIMNHL family of proteins (Mei-P26 and Brat) operates as an uncoupler of RiBi and Tor kinase activities during stem cell differentiation, creating a scenario that deviates from the canonical growth paradigm downstream of Tor activation. In this context, the imbalance between anabolic processes not only alters the growth rate of cells during fate transition but may also create a timer for terminal differentiation. Indeed, often associated with growth and over-proliferation, our results indicate that in a context of suppression of RiBi, activation of the Tor kinase leads to growth suppression instead, ultimately driving cells to terminal commitment. While the molecular mechanisms by which TRIM-NHL activates Tor remains to be determined, the regulation unveiled in this work may have implications in many other developmental transitions. Given the variety of diseases - ranging from cancer to neurodegeneration that are associated with mutations or dysregulation of TRIM-NHL homologs ${ }^{7}$, our work provides a framework for targeted strategies. 


\section{Methods}

\section{Drosophila stocks, genetics, and husbandry}

Drosophila melanogaster stocks and transgenes used: $w^{1118}$ (Lehmann lab stock); UAS-Dcr2, $w^{1118}$; nosPGAL4-NGT40 (BDSC \#25751); P\{bamP-GFP\} transgene ${ }^{29} ; b^{4 a m}{ }^{\Delta 86}, r y, e / T M 3, S b, r y, e^{30} ; y^{l} w^{l} m e i-$ $P 26^{m f s}, ; ; \quad D p(1 ; 4) A 17 / s v^{\text {spa-pol }}$ (BDSC \#25919) ${ }^{31}$; $w^{1118}$; UASp-mei-p26.N (BDSC \#25771); brat ${ }^{11}$; insc-Gal4 (BDSC \#8751) and UAS-mCD8:GFP (BDSC \#5130 and \#5137); UASp-brat ${ }^{32}$; UASraptor-HA (BDSC \#53726); $w^{*}$; FRT 40A (BDSC \#86317); hs-FLP, UAS-mCD8: GFP; tubP-GAL80, FRT 40A; tubP-GAL4 (from BDSC \#44406 and \#84300); w;; [FlyFos020668(Tor29074::2XTY1SGFP-V5-preTEV-BLRP-3XFLAG)dFRT] VK00033 (VDRC \#318201), w;; [FlyFos022619(raptor[16724]::2XTY1-SGFP-V5preTEV-BLRP-3XFLAG)dFRT]VK00033 (VDRC \#318149).

The following UAS-RNAi lines were used: $y^{l} s c^{*} v^{l}$ sev $^{21} ;$ P $\{$ VALIUM20-mCherry\}attP2 (mCherry RNAi; BDSC \#35785); $y^{l} s c^{*} v^{I}$; P\{TRiP.GL01124\}attP40 (mei-P26-shRNA; BDSC \#36855); $y^{l} s c^{*} v^{l}$; P\{TRiP.HMS01121\}attP2 (brat RNAi, BDSC \#34646); $y^{l} s c^{*} v^{l} ;$ P\{TRiP.GL00012\}attP2 (tsc1 RNAi, $\quad$ BDSC \#35144); $y^{l} \quad s c^{*} v^{l}$; P\{TRiP.GL00321\}attP2 (tsc2 RNAi, BDSC \#35401).

Unless stated otherwise, stocks and crosses were maintained on standard propionic food at $25^{\circ} \mathrm{C}$. For rapamycin feeding experiments with adult flies, 200ul 100uM rapamycin (Sigma Aldrich, \#R0395) was added to the top of food at least one day before newly eclosed flies were transferred into the vial. Flies were raised in food containing rapamycin for 3 days at $25^{\circ} \mathrm{C}$ before ovary dissections. For feeding experiments with larvae, 50ul 2mM BMH-21 (Sigma Aldrich, \#SML1183) was added to the top of food for no less than one day before the experiment. Threeday-old larvae grown at room temperature were transferred into the food containing BMH-21 and raised at $29^{\circ} \mathrm{C}$ for three days before brain dissections. For BMH-21 injection in the adult flies, $69 \mathrm{nl} 200 \mu \mathrm{M}$ BMH-21 diluted in the double-stilled water were injected into the abdomen of one-day-old females. Injected flies were raised for five days at $25^{\circ} \mathrm{C}$ before ovary dissections.

\section{Immunofluorescence and antibodies}

Adult ovaries and larval brains were dissected in cold PBS buffer and fixed in PBST (PBS with 0.2\% Triton-X100) containing 4\% Formaldehyde (Thermo Fisher Scientific, \#28908) for 30 minutes. Fixed tissues were rinsed three times with PBST before incubation in blocking buffer (PBS with 5\% goat serum; Sigma Aldrich, \#G9023) overnight at $4^{\circ} \mathrm{C}$. Samples were then incubated with primary antibody diluted in PBST overnight at $4{ }^{\circ} \mathrm{C}$, washed four times with PBST, and incubated with secondary antibodies and DAPI (Sigma Aldrich, \#D9542) diluted in PBST overnight at $4^{\circ} \mathrm{C}$. Samples were washed four times with PBST and mounted in VectaShield medium (Vector Laboratories, \#H1000). Fluorescent images were acquired on a Leica SP8 confocal microscope using a 40X oil objective or a $20 \mathrm{X}$ dry objective. Images were processed using ImageJ (NIH; http://imagej.nih.gov/ij/).

The following antibodies were used for immunofluorescence staining: rat anti-Dpn (Abcam, \#ab195173, 1:200), mouse anti-Fib (Abcam, \#ab4566, 1:200), rabbit anti-HA (Abcam, \#ab9110, 1:100), rat anti-HA (Sigma Aldrich, \#3f10, 1:100), rat anti-GFP (Millipore, \#MAB3580, 1:200), chicken anti-GFP (1:1000), rat anti-RFP (Chromotek, \#5F8, $1: 200$ ), mouse anti- $\alpha$-Spectrin (DSHB, \#3A9, 1:100), mouse anti-Pros (DSHB, \#MR1A, 1:20), rabbit antiphosphorylated-S6 (Dr Aurelio Teleman, 1:200), rabbit anti-Vasa (Dr Ruth Lehmann, 1:5000), rabbit anti-Mei-P26 (Dr. Paul Lasko, 1:1000), goat antimouse Alexa488 (Invitrogen, \#A32723, 1:200), donkey anti-rat Alexa488 (Invitrogen, \#A11006, 1:200), goat anti-rabbit Alexa568 (Invitrogen, \#A11011, 1:200), donkey anti-mouse Cy3 (Jackson ImmunoResearch, \#715-165-150, 1:200), donkey anti-rabbit Alexa647 (Jackson ImmunoResearch, \#711-605-152, 1:200), and goat anti-mouse Alexa647 (Invitrogen, \#A21236, 1:200).

\section{Measurement of global protein synthesis in vivo}

Protein synthesis was detected by the Click-iT® Plus OPP Alexa Fluor 594 Protein Synthesis Assay Kit (Molecular Probes) as previously described ${ }^{5}$. Unless stated otherwise, samples were immediately transferred after dissection to fresh medium containing a 1:400 dilution of Click-iT OPP Reagent (OP-puro $50 \mu \mathrm{M})$. For the experiment in Fig. 1a, dissected ovaries were incubated in medium containing rapamycin for $30 \mathrm{~min}$ before exposure to the Click-iT OPP reagent. Samples were incubated with OPP at room temperature for $30 \mathrm{~min}$, rinsed 3 times with PBS, and fixed with $4 \%$ formaldehyde in PBS for 30 min. After Click-iT® reaction, samples were washed with PBS with $1 \%$ BSA and $0.2 \%$ Triton $\mathrm{X}-100$ for 1 hour and immunostained according to standard procedures. Quantification of OP-Puro fluorescence intensity was performed as previously 
described $^{5}$ using ImageJ. Each experiment was done at least three times.

\section{Nucleolar volume measurement}

The volume of Fibrillarin-stained nucleoli was determined based on Z-stack confocal images using the ' $3 \mathrm{D}$ object counter' plug-in of the ImageJ. Objects on the edge of images were excluded, and threshold and size filters were automatically set. Data in Fig. 2i$\mathrm{j}$ were obtained from three independent ovaries.

\section{RNA sequencing}

About 120 pairs of ovaries were dissected for each sample and immediately stored at $-80^{\circ} \mathrm{C}$ after dissection. Frozen samples were homogenized in TRIzol Reagent (Invitrogen) using an electrical pestle and further disrupted by passing 15 times through a 26-gauge syringe. Total RNA was isolated using TRIzol Reagent (Invitrogen) following the manufacturer's protocol. After RNA quantification using Qubit (Invitrogen), Poly(A)-selected RNAsequencing (RNA-seq) libraries were generated using $2.5 \mu \mathrm{g}$ of purified RNA with the NEBNext ${ }^{\circledR}$ Poly(A) mRNA Magnetic Isolation Module and the NEBNext ${ }^{\circledR}$ Ultra $^{\text {TM }}$ Directional RNA Library Prep Kit for Illumina ${ }^{\circledR}$. Libraries were multiplexed using the NEBNext $^{\circledR}$ Multiplex Oligos for Illumina ${ }^{\circledR}$ and sequenced in single-end, 50-nt-long reads on an Illumina HiSeq 2500. The resulting RNA-sequencing data were first aligned to ribosomal RNA using Bowtie $2^{33}$. Non-rRNA reads were mapped to the Drosophila melanogaster genome (dm6) using $\mathrm{STAR}^{34}$, and transcript abundance was quantified, and differentially expressed genes were identified using Cufflinks ${ }^{35}$. Analyses were performed with two samples, each with two biological replicates.

\section{Climbing assay}

About 20 1-2 days-old flies were transferred into a fresh vial. The height each fly climbed within 20 seconds after knocking down was recorded and quantified. The results represent data collected from six replicas.

\section{Statistics}

All experiments were conducted not less than 3 times independently. Statistical significance ( $p$-value) was tested by applying paired $t$-test method with a $95 \%$ confidence interval or one-way ANOVA. All error bars represent the standard error of the mean (SEM).

\section{Acknowledgments}

We thank A. Teleman for reagents and antibodies; $\mathrm{H}$. Ashe, A. Brand, F. Jiggins, O. Shimmi, the Vienna Drosophila Resource Center, and the Bloomington
Drosophila Stock Center for fly reagents. A. Martinez-Arias and T.J. Samuels for discussions and comments on the manuscript. FKT is a Wellcome Trust and Royal Society Sir Henry Dale Fellow (206257/Z/17/Z) and is supported by the Human Frontier Science Program (CDA-00032/2018).

\section{Author Contributions}

J.G. and F.K.T conceived the idea and designed the experiments. J.G. performed the experiments. F.K.T. analyzed RNA sequencing data. J.G. and F.K.T. wrote the manuscript.

\section{References}

1 Baser, A. et al. Onset of differentiation is posttranscriptionally controlled in adult neural stem cells. Nature 566, 100-104, doi:10.1038/s41586019-0888-x (2019).

2 Blanco, S. et al. Stem cell function and stress response are controlled by protein synthesis. Nature 534, 335-340, doi:10.1038/nature18282 (2016).

3 Buszczak, M., Signer, R. A. \& Morrison, S. J. Cellular differences in protein synthesis regulate tissue homeostasis. Cell 159, 242-251, doi:10.1016/j.cell.2014.09.016 (2014).

4 Sampath, P. et al. A hierarchical network controls protein translation during murine embryonic stem cell self-renewal and differentiation. Cell Stem Cell 2, 448-460, doi:10.1016/j.stem.2008.03.013 (2008).

5 Sanchez, C. G. et al. Regulation of Ribosome Biogenesis and Protein Synthesis Controls Germline Stem Cell Differentiation. Cell Stem Cell 18, 276-290, doi:10.1016/j.stem.2015. 11.004 (2016).

6 Signer, R. A., Magee, J. A., Salic, A. \& Morrison, S. J. Haematopoietic stem cells require a highly regulated protein synthesis rate. Nature 509, 49-54, doi:10.1038/nature13035 (2014).

7 Tocchini, C. \& Ciosk, R. TRIM-NHL proteins in development and disease. Semin Cell Dev Biol 47-48, 52-59, doi:10.1016/j.semcdb.2015. 10.017 (2015).

8 Betschinger, J., Mechtler, K. \& Knoblich, J. A. Asymmetric segregation of the tumor suppressor brat regulates self-renewal in Drosophila neural stem cells. Cell 124, 1241-1253, doi:10.1016/j.cell.2006.01.038 (2006).

9 Lee, C. Y., Wilkinson, B. D., Siegrist, S. E., Wharton, R. P. \& Doe, C. Q. Brat is a Miranda cargo protein that promotes neuronal differentiation and inhibits neuroblast self- 
renewal. Dev Cell 10, 441-449, doi:10.1016/j.devcel.2006.01.017 (2006).

10 Neumuller, R. A. et al. Mei-P26 regulates microRNAs and cell growth in the Drosophila ovarian stem cell lineage. Nature 454, 241-245, doi:10.1038/nature07014 (2008).

11 Chua, B. A., Van Der Werf, I., Jamieson, C. \& Signer, R. A. J. Post-Transcriptional Regulation of Homeostatic, Stressed, and Malignant Stem Cells. Cell Stem Cell 26, 138-159, doi:10.1016/j.stem.2020.01.005 (2020).

12 Teixeira, F. K. \& Lehmann, R. Translational Control during Developmental Transitions. Cold Spring Harb Perspect Biol 11, doi:10.1101/cshperspect.a032987 (2019).

13 Hung, R. J. et al. A cell atlas of the adult Drosophila midgut. Proc Natl Acad Sci U S A 117, 1514-1523, doi:10.1073/pnas.1916820117 (2020).

14 Zhang, Q., Shalaby, N. A. \& Buszczak, M. Changes in rRNA transcription influence proliferation and cell fate within a stem cell lineage. Science 343, 298-301, doi:10.1126/science.1246384 (2014).

15 Llorens-Bobadilla, E. et al. Single-Cell Transcriptomics Reveals a Population of Dormant Neural Stem Cells that Become Activated upon Brain Injury. Cell Stem Cell 17, 329-340, doi:10.1016/j.stem.2015.07.002 (2015).

16 Zismanov, V. et al. Phosphorylation of eIF2alpha Is a Translational Control Mechanism Regulating Muscle Stem Cell Quiescence and Self-Renewal. Cell Stem Cell 18, 79-90, doi:10.1016/j.stem.2015.09.020 (2016).

17 Signer, R. A. et al. The rate of protein synthesis in hematopoietic stem cells is limited partly by 4E-BPs. Genes Dev 30, 1698-1703, doi:10.1101/gad.282756.116 (2016).

18 Spradling, A., Fuller, M. T., Braun, R. E. \& Yoshida, S. Germline stem cells. Cold Spring Harb Perspect Biol 3, a002642, doi:10.1101/cshperspect.a002642 (2011).

19 Liu, G. Y. \& Sabatini, D. M. mTOR at the nexus of nutrition, growth, ageing and disease. Nat Rev Mol Cell Biol 21, 183-203, doi:10.1038/s41580019-0199-y (2020).

20 Pullen, N. et al. Phosphorylation and activation of p70s6k by PDK1. Science 279, 707-710, doi:10.1126/science.279.5351.707 (1998).

21 Romero-Pozuelo, J., Demetriades, C., Schroeder, P. \& Teleman, A. A. CycD/Cdk4 and Discontinuities in Dpp Signaling Activate TORC1 in the Drosophila Wing Disc. Dev Cell
42, 376-387 e375, doi:10.1016/j.devcel.2017. 07.019 (2017).

22 Zielke, N. et al. Fly-FUCCI: A versatile tool for studying cell proliferation in complex tissues. Cell Rep 7, 588-598, doi:10.1016/j.celrep.2014.03.020 (2014).

23 Liu, J., Xu, Y., Stoleru, D. \& Salic, A. Imaging protein synthesis in cells and tissues with an alkyne analog of puromycin. Proc Natl Acad Sci $\begin{array}{llll}U & S & A & 109,\end{array}$ doi:10.1073/pnas.1111561108 (2012).

24 McKearin, D. M. \& Spradling, A. C. bag-ofmarbles: a Drosophila gene required to initiate both male and female gametogenesis. Genes Dev 4, 2242-2251, doi:10.1101/gad.4.12b.2242 (1990).

25 Frank, D. J., Edgar, B. A. \& Roth, M. B. The Drosophila melanogaster gene brain tumor negatively regulates cell growth and ribosomal RNA synthesis. Development 129, 399-407 (2002).

26 Bonnay, F. et al. Oxidative Metabolism Drives Immortalization of Neural Stem Cells during Tumorigenesis. Cell 182, 1490-1507 e1419, doi:10.1016/j.cell.2020.07.039 (2020).

27 Luo, L., Liao, Y. J., Jan, L. Y. \& Jan, Y. N. Distinct morphogenetic functions of similar small GTPases: Drosophila Drac1 is involved in axonal outgrowth and myoblast fusion. Genes Dev 8, 1787-1802, doi:10.1101/gad.8.15.1787 (1994).

28 Peltonen, K. et al. A targeting modality for destruction of RNA polymerase I that possesses anticancer activity. Cancer Cell 25, 77-90, doi:10.1016/j.ccr.2013.12.009 (2014).

29 Chen, D. \& McKearin, D. M. A discrete transcriptional silencer in the bam gene determines asymmetric division of the Drosophila germline stem cell. Development 130, 1159-1170, doi:10.1242/dev.00325 (2003).

30 McKearin, D. \& Ohlstein, B. A role for the Drosophila bag-of-marbles protein in the differentiation of cystoblasts from germline stem cells. Development 121, 2937-2947 (1995).

31 Page, S. L., McKim, K. S., Deneen, B., Van Hook, T. L. \& Hawley, R. S. Genetic studies of mei-P26 reveal a link between the processes that control germ cell proliferation in both sexes and those that control meiotic exchange in Drosophila. Genetics 155, 1757-1772 (2000).

32 Harris, R. E., Pargett, M., Sutcliffe, C., Umulis, D. \& Ashe, H. L. Brat promotes stem cell differentiation via control of a bistable switch that restricts BMP signaling. Dev Cell 20, 72-83, doi:10.1016/j.devcel.2010.11.019 (2011). 
33 Langmead, B. \& Salzberg, S. L. Fast gappedread alignment with Bowtie 2. Nat Methods 9, 357-359, doi:10.1038/nmeth.1923 (2012).

34 Dobin, A. et al. STAR: ultrafast universal RNAseq aligner. Bioinformatics 29, 15-21, doi:10.1093/bioinformatics/bts635 (2013).
35 Trapnell, C. et al. Transcript assembly and quantification by RNA-Seq reveals unannotated transcripts and isoform switching during cell differentiation. Nat Biotechnol 28, 511-515, doi:10.1038/nbt.1621 (2010). 


\section{Extended Data Figure 1}

a

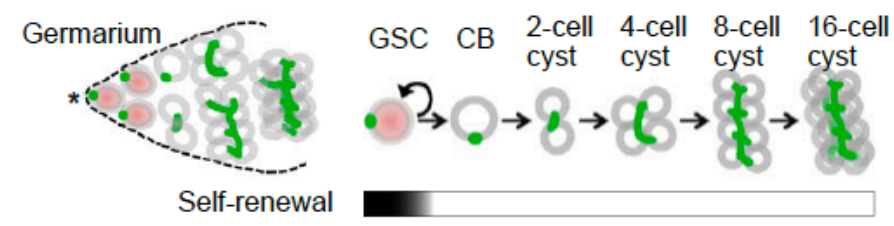

Pro-differentiation (Bam)

Ribosome biogenesis

Global protein synthesis

Tor activity (p-S6)

b

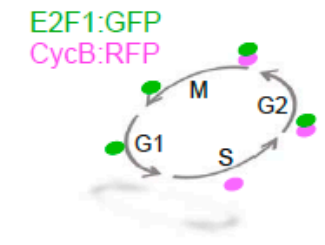

d

The distribution of imaged GSCs $(n=124)$ in cell cycle

C
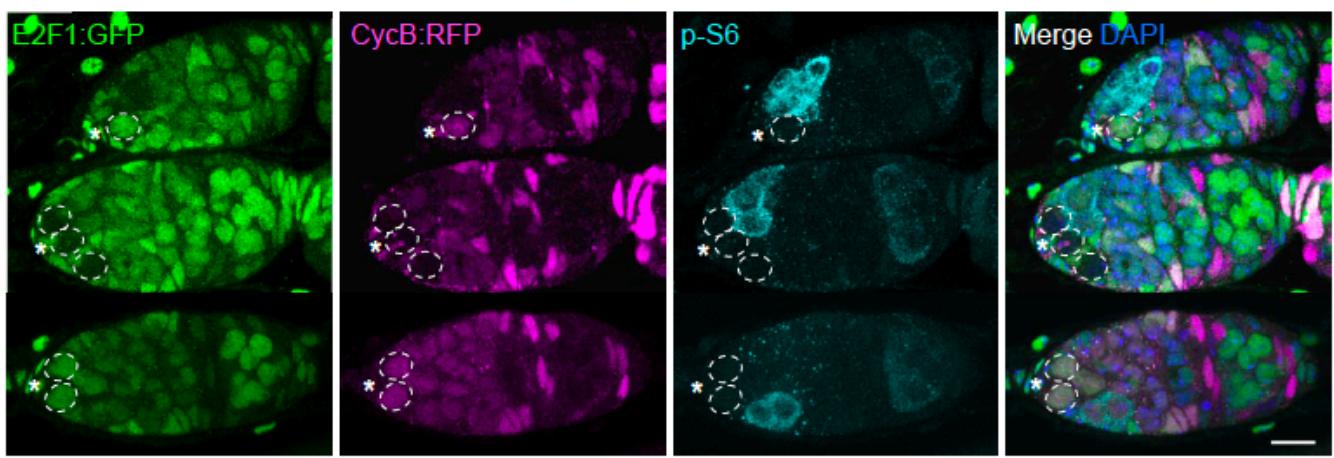

e
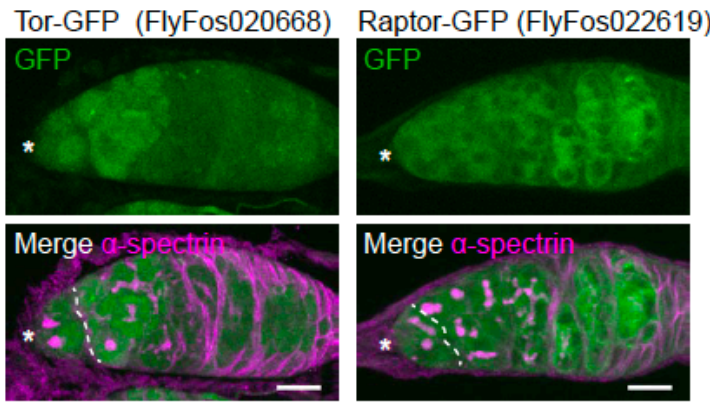

a, Schematics of the structure of germarium and different stages of GSC differentiation. Spectrosomes/fusomes are marked by green. The asterisk indicates GSC niche. GSCs are marked by pink nuclei. $\mathbf{b}$, A schematic showing the rationale of the two-color fly FUCCI system. c, Representative germaria labeled with GFP (E2F1: GFP, green), RFP (CycB:RFP, magenta), p-S6 (Tor activity, cyan), and DAPI (nuclei, blue). Dashed circles mark GSCs. d, Distribution of cell-cycle phase of imaged GSCs $(n=124)$. e, Representative image of germaria of transgenic Tor-GFP (left, FlyFos020668) and Raptor-GFP (right, FlyFos022619) flies labeled with $\alpha$-spectrin (spectrosomes/fusomes, green) and GFP (fusion proteins, green). Dashed lines depict the boundary between GSCs and differentiating cells. The asterisk indicates GSC niche (c, e). Scale bars, $10 \mu \mathrm{m}(\mathbf{c}, \mathbf{e})$. 


\section{Extended Data Figure 2}
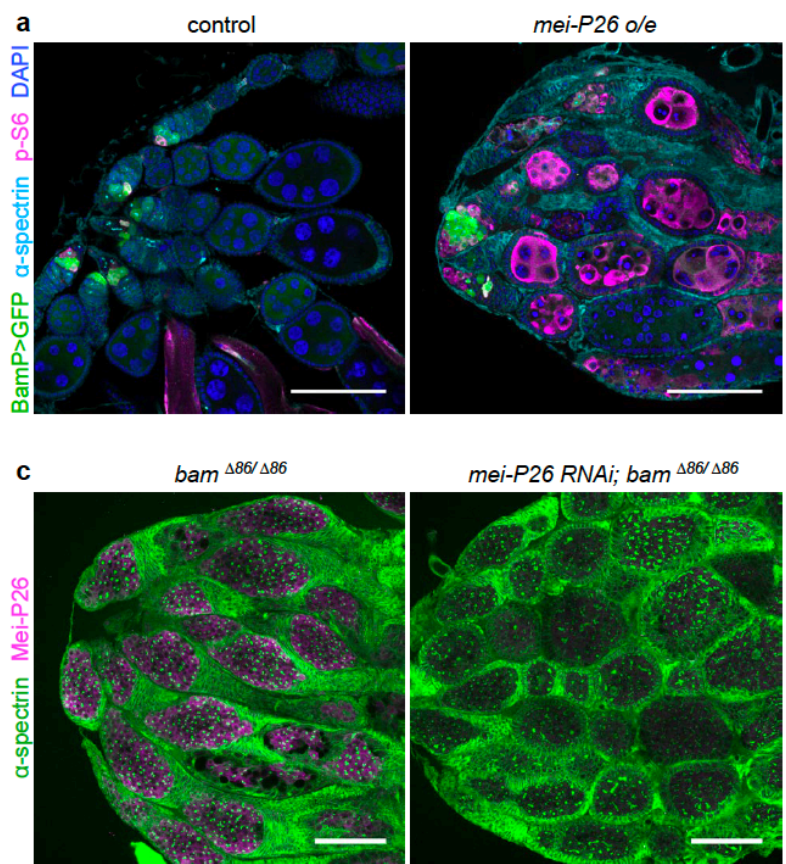

mei-P26 RNAi; bam ${ }^{\Delta 86 / \Delta 86}$

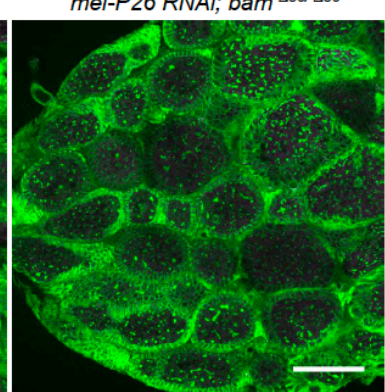

mei-P26 o/e +Rapamycin

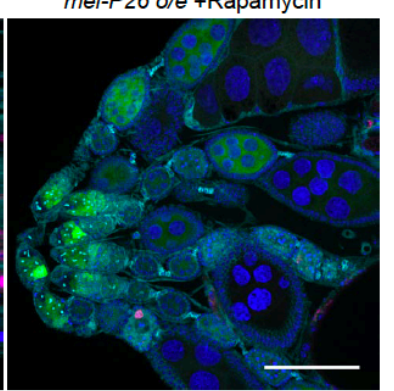

mei-P26 o/e; bam ${ }^{\Delta 86 / \Delta 86}$

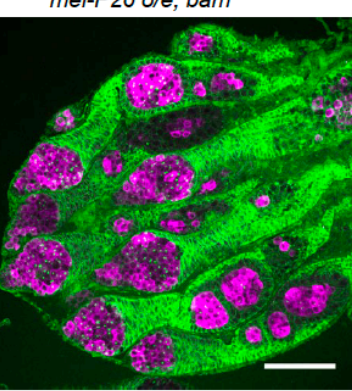

b with GSC $\square$ with CC $\square$ with CB $\square$ empty germarium

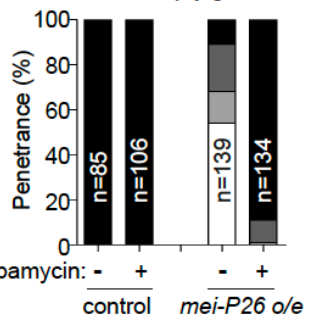

d 2.0 RiBi-related genes

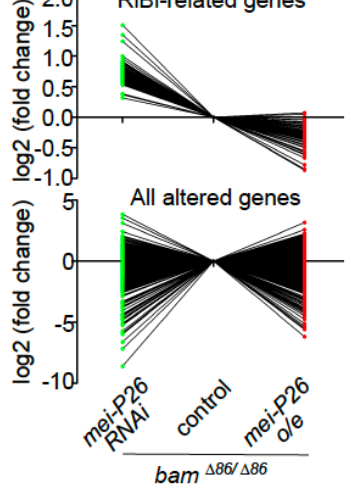

a, Representative ovary images of control (nos-gal4/+) and mei-P26 overexpression (o/e; nos-gal4/UASp-mei$P 26)$ flies with or without rapamycin feeding. Ovaries were stained with GFP (BamP $>$ GFP, differentiating cells, green), p-S6 (Tor activity, magenta), $\alpha$-spectrin (spectrosome/fusome, cyan), and DAPI (nuclei, blue). b, Distribution of germarium phenotypes of control (nos-gal4/+) and mei-P26 overexpression (o/e; nos-gal4/UASpmei-P26) with or without rapamycin feeding. c, Representative ovary images of control (nos-gal4/+), mei-P26 knockdown (nos-gal4/UAS-mei-P26 RNAi), and mei-P26 overexpression (o/e; nos-gal4/UASp-mei-P26) in bam $^{486 /} 486$ background. Samples were stained with $\alpha$-spectrin (spectrosome/fusomes, green) and Mei-P26 (magenta). d, RNA profiling of ovaries of control (nos-gal4/+), mei-P26 knockdown (RNAi, nos-gal4/UAS-mei$P 26$ RNAi), and mei-P26 overexpression (nos-gal4/UASp-mei-P26) in bam ${ }^{486 / 486}$ background. Scale bars, $200 \mu \mathrm{m}$ (a) or $100 \mu \mathrm{m}(\mathbf{c})$. 


\section{Extended Data Figure 3}

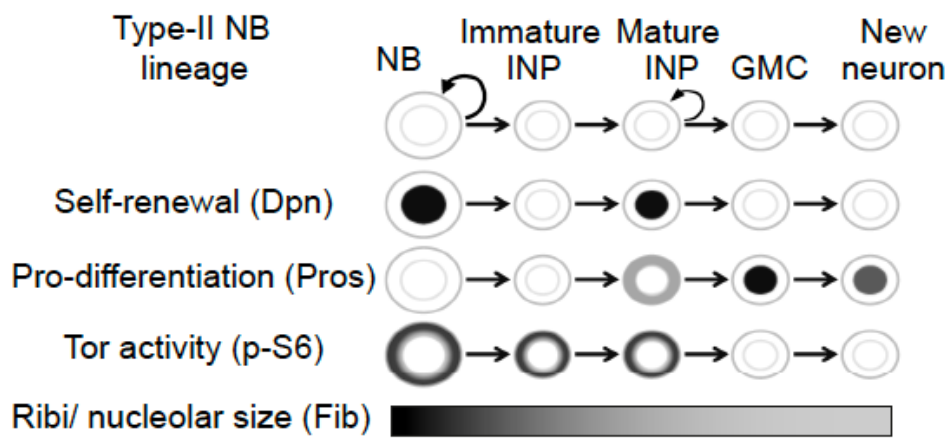

Schematics showing different stages and expression markers during type-II NB differentiation. 
bioRxiv preprint doi: https://doi.org/10.1101/2021.03.31.437822; this version posted April 1, 2021. The copyright holder for this preprint (which was not certified by peer review) is the author/funder, who has granted bioRxiv a license to display the preprint in perpetuity. It is made available under aCC-BY-NC-ND 4.0 International license.

\section{Extended Data Figure 4}

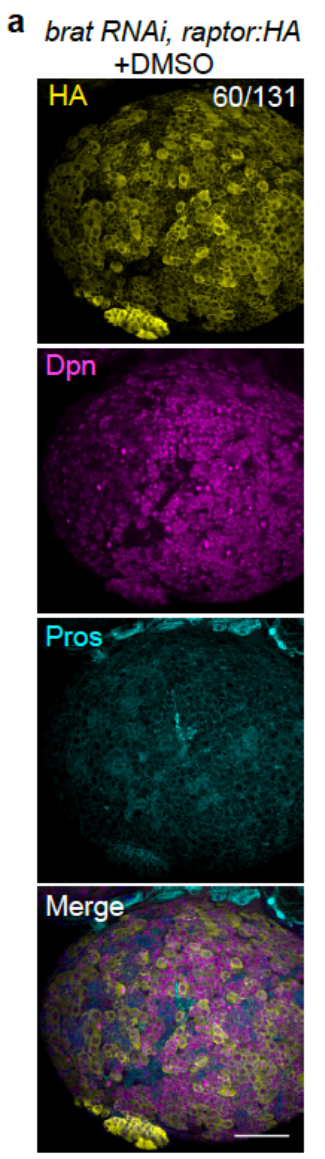

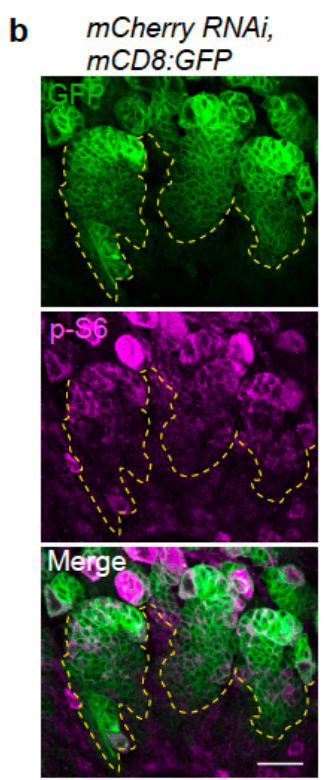

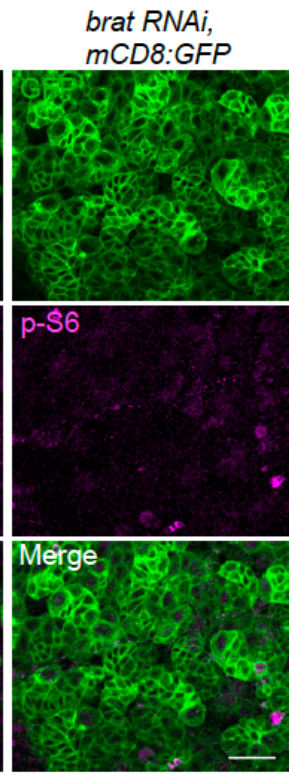

C
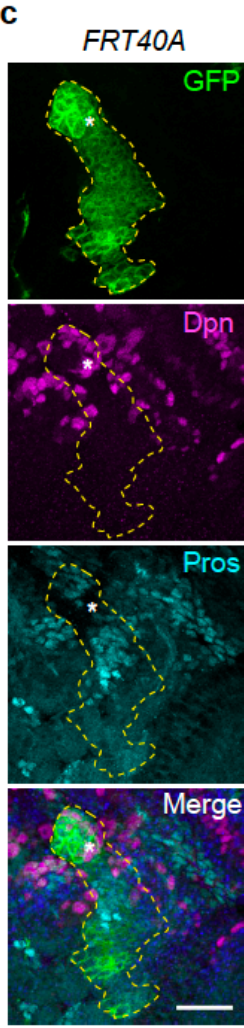

brat RNA

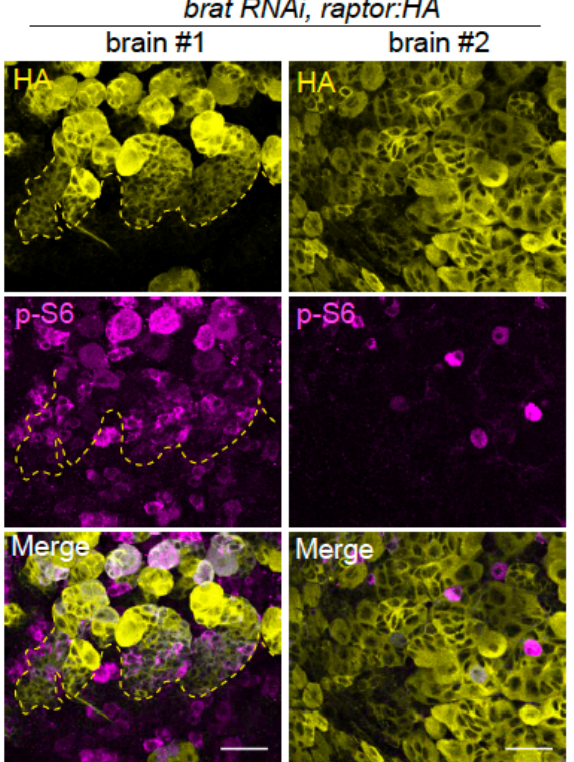

brat ${ }^{11}$ FRT40A, brat ${ }^{11}$ FRT40A,

\section{tsc2 RNAi}
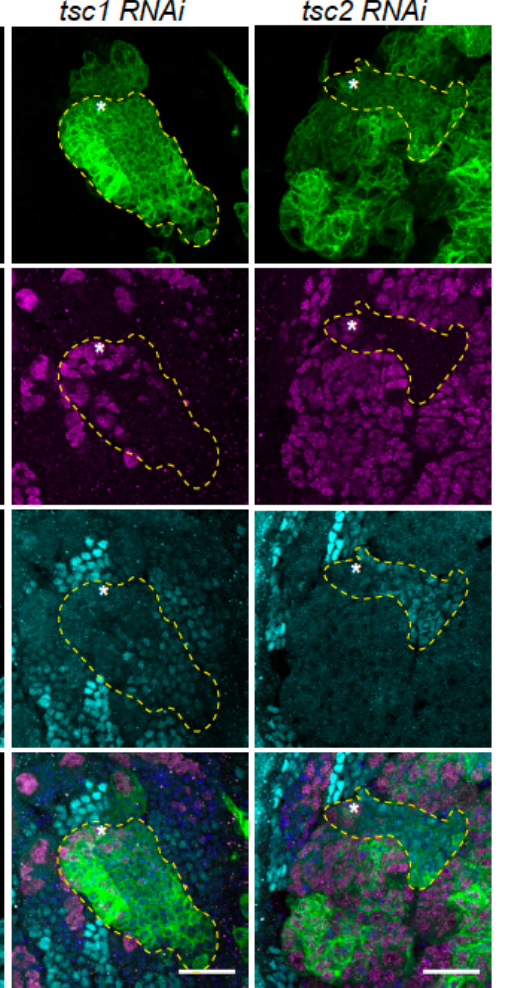

a, Representative brain lobes of brat knockdown (RNAi) together with raptor overexpression (o/e; brat RNAi, raptor:HA) flies (driven by insc-GAL4). Samples were stained with HA (green), Dpn (self-renewal marker, magenta), and Pros (pro-differentiation marker, cyan). The numbers in the upper panel indicate the penetrance of the phenotype. b, Representative confocal images of type-II NB lineages of control ( $m$ Cherry RNAi, $m C D 8$ : GFP), brat knockdown (brat RNAi, mCD8: GFP), and brat knockdown with raptor overexpression (brat RNAi, raptor:HA). Samples were stained with GFP/HA (green) and p-S6 (Tor activity, magenta). Dashed lines depict the border of insc-GAL4 active regions (marked by GFP/HA). c, Representative confocal images of MARCM clonal analysis of control (FRT40A), brat mutant ( rrat $^{11}$ FRT40A), and brat mutant with $t s c 1$ or $t s c 2$ knockdown (brat ${ }^{11}$ FRT40A, tsc1 RNAi or tsc2 RNAi). Samples were stained with GFP (green), Dpn (magenta), Pros (cyan), and DAPI (blue). Dashed lines outline the clonal region. Asterisks indicate type-II NBs within the clone. Scale bars, $50 \mu \mathrm{m}(\mathbf{a})$ or $20 \mu \mathrm{m}(\mathbf{b}, \mathbf{c})$. 


\section{Extended Data Figure 5}

a

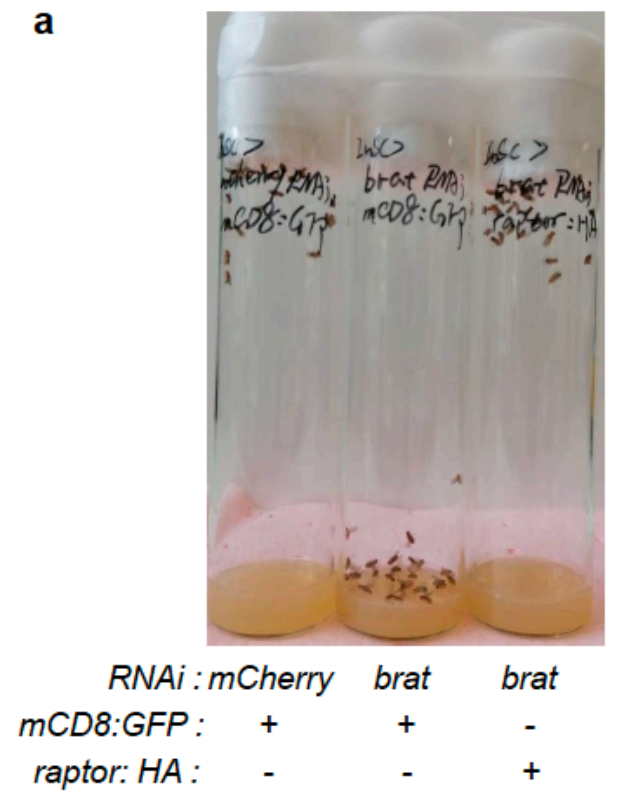

RNAi:mCherry brat brat $m C D 8: G F P$ : raptor: $H A$ : b

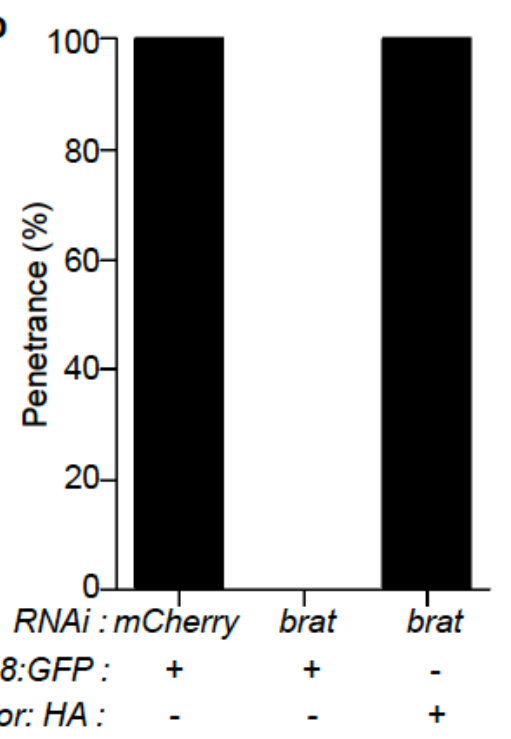

a, Representative images of control ( $m$ Cherry RNAi, $m C D 8: G F P$ ), brat knockdown (brat RNAi, $m C D 8: G F P$ ), and brat knockdown with raptor overexpression (brat RNAi, raptor:HA) adult flies (driven by insc-GAL4). b, Distribution of flies reaching the top of the vial in 20 seconds after knocking for controls ( $m$ Cherry $R N A i$, $m C D 8: G F P$ ), brat knockdown (brat RNAi, $m C D 8: G F P$ ), and brat knockdown with raptor overexpression (brat $R N A i$, raptor:HA). 
bioRxiv preprint doi: https://doi.org/10.1101/2021.03.31.437822; this version posted April 1, 2021. The copyright holder for this preprint (which was not certified by peer review) is the author/funder, who has granted bioRxiv a license to display the preprint in perpetuity. It is made available under aCC-BY-NC-ND 4.0 International license.

\section{Extended Data Figure 6}

\section{a}
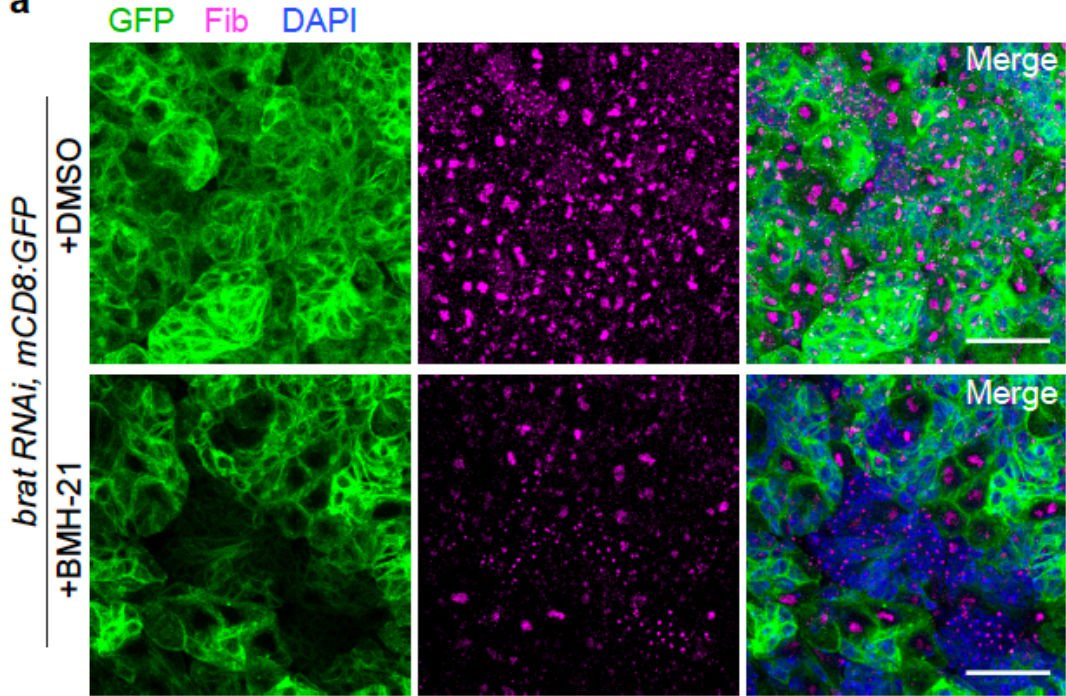

b

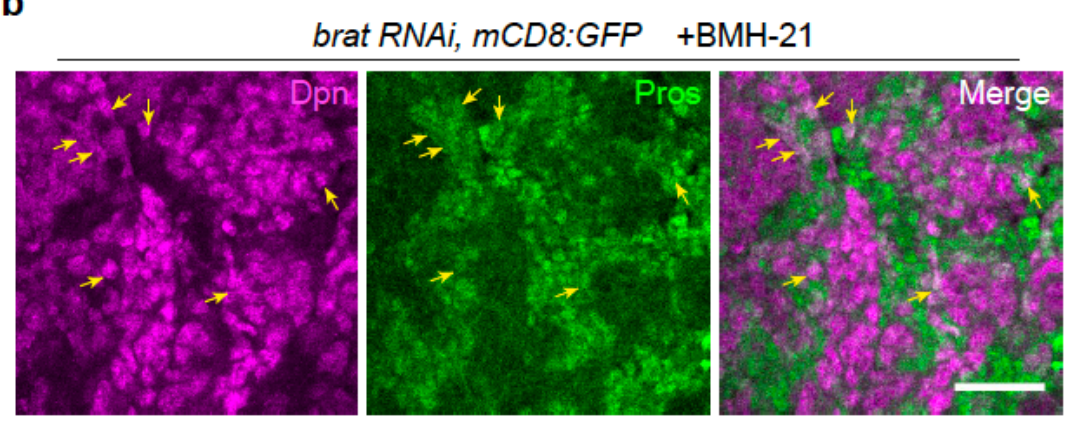

a, Representative confocal images of brat knockdown (brat RNAi, mCD8:GFP) flies (driven by insc-GAL4) with or without BMH-21 feeding, stained with GFP (green), Fib (nucleoli, magenta), and DAPI (blue). b, Representative confocal images of brat knockdown (brat RNAi, mCD8:GFP) flies (driven by insc-GAL4) with BMH-21 feeding, marked by Pros (magenta) and Dpn (green). Arrows indicated the cells co-expressing Dpn and Pros markers. Scale bars, $20 \mu \mathrm{m}$. 